\title{
GROUPS OF RATIONAL TRANSFORMATIONS IN A GENERAL FIELD*
}

BY

\author{
LEWIS IRVING NEIKIRK
}

\section{Introduction.}

Groups of linear transformations of a single variable of both finite and infinite orders are well known, but the only known examples of non-linear rational transformation groups in one variable are those given by the following writers: Hermite, BetTi, and others have investigated special quantics, known as substitution quantics, with coefficients taken with respect to a prime modulus $(p)$, which define substitutions on a set of residues $(\bmod p)$ and generate finite groups $(\bmod p)$. Substitution quantics with coefficients in a Galois field have been investigated by Dickson in his dissertation, $\dagger$ where the reader will find a complete bibliography of the subject.

The object of this paper is to find all non-linear groups of rational transformations of a single variable. It is proved in $\S 1$ that these groups of transformations define substitution groups on the roots of an equation $f(x)=0$. They are a two-fold generalization of substitution quantics and form finite groups $(\bmod f(x))$. Section 2 is devoted to finding these transformations and section 3 to the conditions for the existence of such transformations in a general field $F$. The other articles apply and extend these results.

\section{§1. General developments.}

Consider a group $G$ of rational integral transformations

$$
\begin{gathered}
T_{i} \equiv\left[x: \phi_{i}(x)\right], \\
\phi_{i}(x)=\sum_{j=0}^{j=m_{i}} \alpha_{i j} x^{m_{i}-j}
\end{gathered}
$$$$
\left(\alpha_{i 0} \neq 0\right) \text {, }
$$

where the coefficients $\alpha_{i j}$ are elements of a general field $F$ and the quantity $x$ belongs to a set $X_{i}$ in a field $F^{\prime}$ containing $F$. It is assumed that at least one $m_{i}$ exceeds unity, so that the group is not linear.

* Presented to the Society (Chicago), April and December, 1909.

$\dagger \mathrm{L}$. E. Dickson, The analytical representation of substitutions on a power of a prime number of letters, etc., Annals of Mathematics, ser. 1., vol. 11 (1896), pp. 65-120, 161-183. 
Let $T_{i}\left(X_{i}\right)=X_{i}^{\prime}$. Then*

$$
\begin{aligned}
& X_{i} \equiv X_{i}^{\prime}, \text { for every } i . \\
& X_{i} \equiv X_{i^{\prime}} \equiv X, \text { for every } i \text { and } i^{\prime} .
\end{aligned}
$$

(a) Since $T_{i}^{2}$ is in $G, X_{i}^{\prime}$ is a subset of $X_{i}$, and since $T_{i}^{-2}$ is in $G, X_{i}$ is a subset of $X_{i}^{\prime}$. Therefore $X_{i} \equiv X_{i}^{\prime}$.

(b) $X_{i}$ must be a subset of $X_{i^{\prime}}$ since $T_{i^{\prime}} T_{i}$ is in $G$, and $X_{i^{\prime}}$ must be a subset of $X_{i}$ since $T_{i} T_{i^{\prime}}$ is in $G$. Therefore $X_{i} \equiv X_{i^{\prime}} \equiv X$.

Since $T_{i}$, of degree $m_{i}>1$, has an inverse in $G$, let $T_{i}^{-1}=T_{i^{\prime}}$. Then

whence

$$
T_{i} T_{i^{\prime}} \equiv[x: x]=\left[x: \phi_{i}\left\{\phi_{i^{\prime}}(x)\right\}\right] \text {, }
$$

$$
\phi_{i}\left\{\phi_{i^{\prime}}(x)\right\}=x,
$$

so that $x$ satisfies an equation of degree $m_{i} m_{i^{\prime}}>1$, the leading coefficient being $\alpha_{i 0} \alpha_{i^{\prime} 0} \neq 0$.

Therefore the elements of the set $\boldsymbol{X}$ are roots of an equation rational in $\boldsymbol{F}$.

Let $X=\left(x_{1}, x_{2}, x_{3}, \ldots, x_{n}\right)$ be a set whose elements are the roots of an equation,

$$
f(x)=\sum_{r=0}^{r=n} a_{r} x^{n-r}=0,
$$

with the coefficients in $F$ and having no double root.

All the transformations reduce $(\bmod f(x))$ to degree $n-1$ or less. $\dagger$

Let $T_{i}$ change $X$ according to the scheme

$$
\left(\begin{array}{c}
x_{1} x_{2} \cdots x_{n} \\
x_{i_{1}} x_{i_{2}} \cdots x_{i_{n}}
\end{array}\right) .
$$

If any root is repeated in the lower line, $T_{i}$ will not have an inverse in the group $G$. Therefore the lower line is a permutation of the upper line and $T_{i}$ defines a substitution on the roots of $f(x)=0$. Hence we have proved

THEOREM I. The only non-linear groups of rational integral transformations on one variable are finite groups taken modulo $f(x)$ which define substitution groups on the roots of the equation $f(x)=0 . \ddagger$

§ 2. Determination of the transformation corresponding to a given substitution. §

Given a substitution on the roots of $f(x)=0$,

$$
S_{i}=\left(\begin{array}{c}
x_{1} x_{2} \cdots x_{n} \\
x_{i_{1}} x_{i_{2}} \cdots x_{i_{n}}
\end{array}\right),
$$

* BURNsIDE (Theory of Groups, p. 12) makes use of property (a) without explicit mention in the proof that if $A_{-1}$ is the inverse of $A_{\text {, then }} A$ is the inverse of $A_{-1}$.

† H. W EBER, Lehrbuch der Algebra, vol. I, p. 170.

$¥$ The actual existence of these groups will be established in the next two articles.

\& L. E. Dickson, Dissertation, l. c. 
we seek the corresponding transformation $T_{i}$. We have the $n$ linear equations

$$
x_{i t}=\phi_{i}\left(x_{t}\right)=\sum_{j=0}^{j=n-1} a_{i j} x_{t}^{n-1-j} \quad(t=1,2, \cdots, n)
$$

between the $n$ coefficients $\alpha_{i v}$. From these

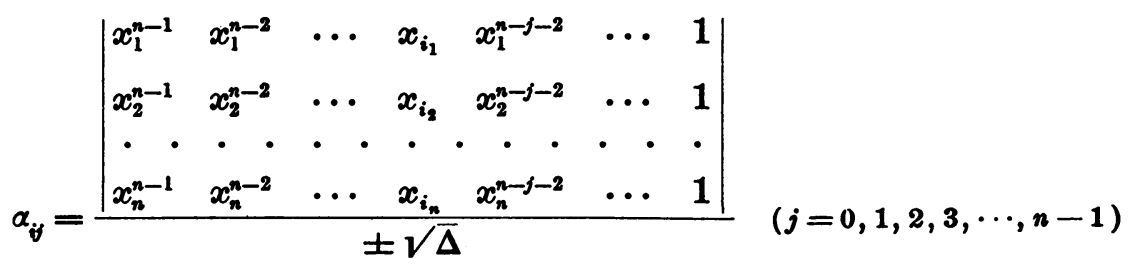

where $\Delta$ is the discriminant of $f(x)$, so that

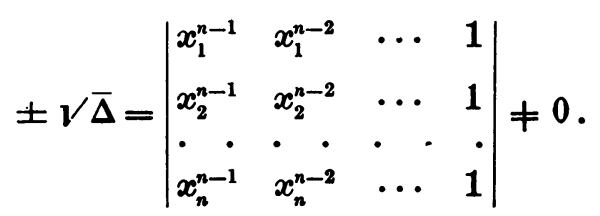

We can also determine $T_{i}$ by the Lagrangian interpolation formula

$$
\phi_{i}(x)=\sum_{t=1}^{t=n} \frac{x_{i_{t}} f(x)}{\left(x-x_{t}\right) f^{\prime}\left(x_{t}\right)}, \quad f(x)=\left(x-x_{1}\right)\left(x-x_{2}\right) \cdots\left(x-x_{n}\right) .
$$

The coefficients of $\phi_{i}$ determined by either of these two methods are not necessarily contained in the general field $F$.

§ 3. Condition for transformations with coefficients in $F$.

Theorem II. The necessary and sufficient condition for the existence of the transformation $T$ with coefficients in the field $F$ on the roots of the equation $f(x)=0$ with coefficients in $F$ is that the substitution $S$ be permutable with every substitution of the Galois group of $f(x)=0$ for $F$.

Let

$$
S=\left(\begin{array}{l}
x_{t} \\
x_{t S}
\end{array}\right)
$$$$
(t=1,2, \cdots, n) \text {. }
$$

Determine $\phi(x)$ by means of one of the two methods given in section 2. We have the equations.

$$
x_{t s}=\phi\left(x_{t}\right) \quad(t=1,2,3, \cdots, n) .
$$


(1) Proof that condition is necessary. The coefficients of $\phi$ are in $F$ by hypothesis. Hence we may apply to (3) the substitutions $R$ of the Galoisian group.* Hence

But, by (3),

$$
x_{t S R}=\phi\left(x_{t R}\right)
$$

$$
x_{t R S}=\phi\left(x_{t R}\right) \text {. }
$$

Hence $x_{t R S}=x_{t S R}$ for every $t$, and thus $R S=S R$.

(2) Proof that the condition is sufficient. By hypothesis, $R S=S R$ for every $R$ in the Galoisian group.

Let $x_{t R}=x_{p}$. Then $x_{t S R}=x_{t R S}=x_{p s}$. Hence if $R$ replaces $x_{t}$ by $x_{p}$ it replaces $x_{t s}$ by $x_{p s}$. In $\S 2, x_{1 s}, \ldots, x_{n s}$ were denoted by $x_{i_{1}}, \ldots, x_{i_{s}}$. Hence if $R$ replaces $x_{t}$ by $x_{p}$, it replaces $x_{i}$ by $x_{i,}$. Hence the coefficients of $\phi$ given by equation (2) are unaltered by $R$ and thus belong to $F$.

\section{§4. The representation of substitutions:}

The substitution

$$
S_{i} \equiv\left(\begin{array}{llll}
x_{1} & x_{2} & \cdots & x_{n} \\
x_{i_{1}} & x_{k_{2}} & \cdots & x_{k_{k}}
\end{array}\right)
$$

can be represented by the transformation

where

$$
T_{i} \equiv\left[x_{t}: x_{\phi(t)}\right],
$$

$$
\phi_{i}(t)=\sum_{j=0}^{j=n} \frac{i_{j} f(t)}{(t-j) f^{\prime}(j)}, \quad f(t)=(t-1)(t-2) \cdots(t-n) .
$$

We may also determine the coefficients of

from the $n$ linear equations

$$
\phi_{i}(t)=\sum_{j=0}^{j=n-1} a_{i j} t^{n-1-j}
$$

The results are

$$
\phi_{i}(t)=i_{t} \quad(t=1.2,3, \cdots, n) .
$$

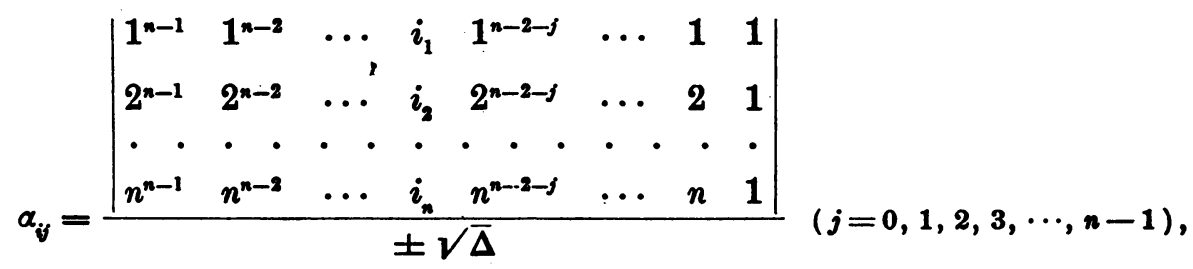

* The theorems used here are known as properties $A$ and $B$ of the Galois group. See Dicksos, Introduction to the theory of algebraic equations, p. 53. 
where

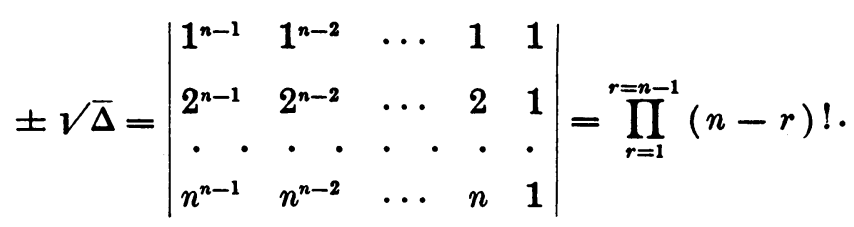

1. Let $n=3$ and

\section{§5. Special examples.}

Then

$$
S_{1}=\left(x_{1} x_{2} x_{3}\right), \quad S_{2}=\left(x_{1} x_{2}\right) \text {. }
$$

$$
\begin{gathered}
f(t)=t^{3}-6 t^{2}+11 t-6, \quad \phi_{1}(t)=-\frac{3}{2} t^{2}+\frac{11}{2} t-2, \\
\phi_{2}(t)=\frac{3}{2} t^{2}-\frac{11}{2} t+6 .
\end{gathered}
$$

These define the symmetric group on three letters.

2. Let $n=4$ and

Then

$$
S_{1}=\left(x_{1} x_{2} x_{3} x_{4}\right), \quad S_{2}=\left(x_{1} x_{2}\right)\left(x_{3} x_{4}\right), \quad S_{3}=\left(x_{1} x_{2} x_{3}\right) .
$$

$$
\begin{gathered}
f(t)=t^{4}-10 t^{3}+35 t^{2}-50 t+24, \quad \phi_{1}(t)=-\frac{2}{3} t^{3}+4 t^{2}-\frac{19}{3} t+5, \\
\phi_{2}(t)=-\frac{4}{3} t^{3}+10 t^{2}-\frac{65}{3} t+15, \quad \phi_{3}(t)=\frac{4}{3} t^{3}-\frac{19}{2} t^{2}+\frac{12}{6} \frac{1}{6} t-10 .
\end{gathered}
$$

These define the symmetric group on four letters.

\section{§6. Rational fractional transformations.}

The results of the previous articles can be extended to rational fractional transformations.

Consider a group $G$ of transformations

where

$$
T_{i} \equiv\left[x: \psi_{i}(x)\right]
$$

$$
\psi_{i}(x)=\frac{\phi_{i}(x)}{\theta_{i}(x)}, \quad \phi_{i}(x)=\sum_{j=0}^{j=m_{i}} \alpha_{i j} x^{m_{i}-j}, \quad \theta_{i}(x)=\sum_{j=0}^{j=n_{i}} \beta_{i j} x^{n_{i}-j}
$$

$\alpha_{i 0} \neq 0, \beta_{i 0} \neq 0$, while $\phi_{i}(x)$ and $\theta_{i}(x)$ have no common factor and at least one of the degrees $m_{i}, n_{i}$ exceeds unity.

The coefficients $\alpha_{i j}$ and $\beta_{i j}$ are elements of a general field $F$ and the quantity $x$ belongs to a set $X$ in a field $F^{\prime}$. As before, these transformations are associative and have the closure property. If $T_{i}$ and $T_{i^{\prime}}$ are inverses

and we have

$$
T_{i} T_{i^{\prime}} \equiv[x: x]=\left[x: \psi_{i}\left\{\psi_{i^{\prime}}(x)\right\}\right]
$$

$$
\psi_{i}\left\{\psi_{i^{\prime}}(x)\right\}=x
$$


This is either (a) an equation of condition, $f(x)=0$, or (b) an identity.

(a) In this case the transformations reduce $[\bmod f(x)]$ to the integral form considered in the first part of the paper.*

(b) In this case,

$$
y=\frac{\phi_{i}(x)}{\theta_{i}(x)} \quad \text { gives } \quad x=\frac{\phi_{i^{\prime}}(y)}{\theta_{i^{\prime}}(y)}
$$

therefore to each $y$ there is only one $x$ and therefore $\phi_{i}(x)$ and $\theta_{i}(x)$ are linear. Case $(b)$ is therefore excluded.

\section{§ 7. Representation of products of substitutions.}

Consider any $k$ substitutions $R_{j}$ of order $r_{j}(j=1,2, \ldots, k)$ on the $n$ roots of $f(x)=0$.

Take the products of powers of these substitutions of the form $\dagger$

The number of these products is

$$
S_{i}=R_{1}^{y^{(i)}} R_{2}^{y^{(i)}} \ldots R_{k}^{y^{(i)}} \equiv\left[\begin{array}{cccc}
x_{1} & x_{2} & \cdots & x_{n} \\
x_{i_{1}} & x_{i_{2}} & \cdots & x_{i_{n}}
\end{array}\right] .
$$

$$
r=\prod_{j=1}^{j=k} r_{j}
$$

and $i$ will have the range $1,2, \ldots, r$.

When the basic substitutions $R_{j}(j=1,2, \ldots, k)$ are given, $S_{i}$ will be determined by the exponents $y_{1}^{(i)}, y_{2}^{(i)}, \ldots, y_{k}^{(i)}$.

It is possible to represent all these substitutions by the transformations

$$
T_{i} \equiv\left[x: \phi\left(x ; y_{1}^{(i)}, y_{2}^{(i)}, \ldots, y_{k}^{(i)}\right)\right]
$$

where $\phi$ is determined by the generalized Lagrangian interpolation formula

and

$$
\phi\left(x ; y_{1}, y_{2}, \ldots, y_{k}\right)=\sum_{l=1}^{l=n} \sum_{j=1}^{j=r} \frac{x_{j_{l}} f(x)}{\left(x-x_{l}\right) f^{\prime}\left(x_{l}\right)} \prod_{p=1}^{p=k} \frac{\theta_{p}\left(y_{p}\right)}{\left(y_{p}-y_{p}^{(j)}\right) \theta_{p}^{\prime}\left(y_{p}^{(j)}\right)}
$$

$$
f(x)=\prod_{t=1}^{t=n}\left(x-x_{t}\right), \quad \theta_{p}\left(y_{p}\right)=\prod_{s=1}^{s=r}\left(y_{p}-y_{p}^{(s)}\right)
$$

When any particular set of $y$ 's as $\left(y_{1}^{(i)}, y_{2}^{(i)}, \ldots, y_{k}^{(i)}\right)$ are substituted in the above it reduces to the regular Lagrangian formula and gives the $\phi_{i}(x)$ used in first part of this paper and therefore $T_{i}$. The function $\phi$ is a rational integral

* H. WeBER, Lehrbuch der Algebra, vol. 1, p. 170.

$\dagger$ No two sets $\left(y_{1}^{(i)}, y_{2}^{(i)}, \cdots, y_{k}^{(i)}\right)$ are alike but no assumption is made concerning the corresponding $S_{i}$. 
function of $x$ whose coefficients are rational integral functions of the $k$ parameters $y_{1}, y_{2}, \ldots, y_{k}$. The numerical coefficients will be contained in the field $F$. when $S_{i}$ fulfills the conditions in Theorem II for every value of $i$.

Any set of substitutions $S_{i}(i=1,2, \ldots, r)$ where each substitution is characterized by a particular set of values $y_{1}^{(i)}, y_{2}^{(i)}, \ldots, y_{k}^{(i)}$ of the $k$ parameters $y_{1}, y_{2}, \cdots, y_{k}$ can be represented by transformations $T_{i}$ determined as above. It is therefore possible to represent * an entire group of transformations by a single formula ( $( \pm)$.

UNIVERSITY OF ILLINOIS, URBANA, ILLINOIS.

* Some of the transformations may be repeated. 